\title{
Potencialidades del análisis de redes para el estudio de las migraciones ${ }^{1}$
}

Potentialities of social network analysis for migration studies

\author{
Verónica de Miguel Luken \\ Universidad de Málaga \\ vdmiguel@uma.es (ESPAÑA)
}

Recibido: 01.112018

Aceptado: 25.02 .2020

\section{RESUMEN}

El presente trabajo examina las principales aportaciones realizadas a la investigación de la inmigración extranjera aplicando el análisis de redes sociales, fundamentalmente en el contexto español. Para ello, se emplea una doble perspectiva. Por un lado, se atiende a la aproximación metodológica particular utilizada en la recogida de datos y en las técnicas estadísticas usadas para su análisis. Por otro, se ubican los trabajos en los ejes temáticos principales identificados. Previamente, se contextualiza la cuestión en el debate más general sobre redes migratorias y capital social, se presentan algunos conceptos sobre el análisis de redes y se proporcionan algunas claves sobre la recogida de datos reticulares y las técnicas más apropiadas para su explotación. Por último, se sugieren las fortalezas de esta perspectiva metodológica y las limitaciones a las que se enfrenta su utilización.

\section{PALABRAS CLAVE}

Metodología, análisis de redes sociales, recogida de datos, estado de la cuestión, inmigración extranjera.

${ }^{1}$ Este artículo se elabora en el marco del proyecto I+D+i del Programa FEDER Andalucía 2014-2020 financiado por la Unión Europea (UMA18-FEDERJA-103) y del proyecto del Plan Nacional I+D+i (CSO2017-86349-P) financiado por el Ministerio de Ciencia, Innovación y Universidades. 


\begin{abstract}
In this paper, the main contributions to the research on foreign migration through the application of social network analysis are examined, especially for the Spanish context. A dual perspective is used. On one hand, the focus is on the specific methodological approach applied for the data collection and the statistical techniques chosen for the analysis. On the other hand, the works are classified according to a proposed thematic division. Previously, the topic is framed in the more general debate about migration networks and social capital, some concepts on network analysis are introduced and some key facts about network data collection and the appropriate techniques for their exploitation are discussed. Finally, the strengths and the limitations of this methodological approach are suggested.
\end{abstract}

\title{
KEY WORDS
}

Methodology, social network analysis, data collection, state of the art, foreign immigration.

\section{INTRODUCCIÓN}

El concepto de redes se ha utilizado en la literatura académica de formas diversas en relación con el estudio de las migraciones, pero normalmente vinculado al capital social de los colectivos inmigrantes y a la idea de la inercia de los flujos migratorios en forma de cadenas. Sin embargo, en estos enfoques lo habitual no ha sido la aplicación de una metodología específica de análisis de redes sociales (ARS) en el sentido más estricto y se ha realizado con frecuencia un uso metafórico de las redes (Ávila 2008; Bilecen, Gamper y Lubbers 2018). Esta metodología, que se basa en el estudio de los vínculos entre actores y no se centra únicamente en sus características individuales, que es la aproximación de las técnicas estadísticas más tradicionales, requiere de datos más completos y difíciles de obtener, de ahí que se encuentre un menor número de investigaciones que adopten dicha perspectiva.

Es lógico, por tanto, que en ocasiones se produzca una cierta confusión en la terminología empleada. El propósito de este artículo es, por una parte, establecer un punto de partida a partir de la clarificación de algunas ideas relacionadas con el análisis de redes, de forma sucinta. Por otra, describir los ejes metodológicos y temáticos del ARS más comúnmente aplicados en la resolución de preguntas de investigación que incorporan su interés por poblaciones migrantes, y ofrecer ejemplos de sus posibles combinaciones a través de una discusión de sus potencialidades y limitaciones.

Dada la imposibilidad de ser completamente exhaustivos en la presentación de los trabajos empíricos sobre análisis de redes aplicado a las migraciones, por 
los límites de extensión de este artículo, se han debido adoptar ciertos criterios para acotar la exploración de aportaciones a la cuestión. Se dará prioridad fundamentalmente a los estudios que abordan el contexto español, aunque se mencionen algunos trabajos muy recientes o de obligada referencia sobre otros lugares. El auge de la producción de investigaciones en migraciones y redes en España discurre en paralelo a la propia cronología de los flujos inmigratorios hacia este país, respaldado por el continuo desarrollo de técnicas estadísticas y herramientas informáticas, cada vez más sofisticadas, para la recogida de datos y su análisis posterior. Esta mayor productividad concentrada en los últimos diez o quince años ha implicado una menor atención a los procesos migratorios internos dentro de nuestras fronteras, por lo que los colectivos de interés suelen referirse a los de origen extranjero. Asimismo, como es bien sabido, la mayor facilidad que conlleva el estudio de la inmigración frente al de la emigración, supone la focalización generalizada hacia las poblaciones foráneas desde el punto de vista de la sociedad receptora.

Finalmente, aunque existe amplia bibliografía sobre las redes sociales de la población de origen inmigrante analizadas desde la aplicación de técnicas cualitativas, este texto se centra fundamentalmente en investigaciones llevadas a cabo desde una perspectiva cuantitativa o mixta que hayan aplicado ARS. Además, se dejarán de lado las publicaciones referidas a redes bimodales, cuyo estudio ha sido bastante exiguo (Gaete Fiscella 2014) o a las más recientes sobre las redes sociales virtuales (Gualda y Rebollo Díaz 2016).

El artículo sigue el siguiente esquema. En primer lugar, se dedica un apartado a la idea de redes y capital social en sentido amplio, como fundamento teórico de los estudios en los que se aplica análisis de redes. Seguidamente se procede a la distinción, en dos apartados diferentes, entre las redes sociocéntricas y las redes egocéntricas. Se proporcionará una breve explicación de las mismas si bien, por limitaciones de espacio, esta se orientará básicamente a la recogida de datos, aunque se mencionen las técnicas de análisis más comúnmente empleadas. En cada uno de los apartados, se aportarán ejemplos en función de la metodología utilizada y de los grandes ejes temáticos alrededor de los que se articulan. Se concluirá con alguna reflexión acerca de las ventajas y desventajas del ARS y algunas propuestas de líneas futuras de investigación en base a las carencias detectadas en la bibliografía analizada.

\section{REDES MIGRATORIAS COMO METÁFORA Y CAPITAL SOCIAL}

\subsection{Redes y cadenas migratorias}

Aunque no usaran la metodología propia del ARS, muchos estudios sobre redes se convirtieron en fuentes de inspiración para las investigaciones posteriores que intentaron conjugar todo el bagaje teórico con las nuevas técnicas metodológicas. En estos trabajos no se recogían datos reticulares, sobre las relaciones entre los miembros de las redes y, a menudo, ni siquiera información detallada 
sobre quiénes las conformaban, sino que se abordaba de una forma más vaga la existencia o no de contactos en el destino o de comunidades connacionales en la sociedad receptora.

En ellos, el concepto de redes migratorias se utilizó fundamentalmente en dos sentidos:

1) como cadena migratoria, con toda la idea de linealidad que conlleva, de eslabones que preceden a otros eslabones, como contactos que orientan, si no tanto en la decisión a emigrar (de la que pueden ser en cualquier caso acicates), sí al menos en la elección del destino. Compatriotas o paisanos, padroni y paessani para los italianos, zemiliacki para los rusos..., los pioneros actúan como reclamo y apoyo para los que siguen, y estos, a su vez, pueden continuar con el mismo rol hacia quienes vienen detrás (MacDonald y MacDonald 1964; Massey et al. 1993; Gurak y Caces 1992; Maya Jariego 2004, 2006; Requena Santos 2008), si bien, con el paso del tiempo, las estructuras de la población de un mismo origen cambian en el destino. Pedone (2003), por ejemplo, observa como los entramados de solidaridad entre la población ecuatoriana en una primera etapa de flujos intensos se jerarquiza con posterioridad dando lugar a estructuras más verticales;

2) como entramados sociales que reducen los costes derivados de la emigración, tanto en lo emocional (aportan compañía en el contexto extraño), como en lo instrumental (proporcionando información sobre el nuevo lugar de residencia, sobre las oportunidades de trabajo, facilitando el primer acceso a la vivienda o incluso recursos de tipo económico o material) (Massey et al. 1993; Gurak y Caces 1992, Maya Jariego 2004, 2006; Requena Santos 2008). En los espacios públicos, en las reuniones y lugares de encuentro se busca a los connacionales, con quienes no existe, entre otros obstáculos, la barrera del idioma (Bojarczuk y Mühlau 2018). El posible riesgo de estas mallas de paisanaje en el destino (Maya Jariego 2006) es el de retardar la integración relacional con la población autóctona, el de producir un encapsulamiento en enclaves étnicos que, además, pueda ejercer un mayor control normativo del deseado en la población extranjera por parte de sus compatriotas (Gurak y Caces 1992, Maya Jariego 2004, Portes y Sensenbrenner 1993).

\subsection{Capital social y migraciones}

En ambos enfoques de las redes sociales como maquinaria que incita a la movilidad y facilita la primera adaptación de la persona inmigrada, subyace la noción de capital social. La aclaración que ofrece Alejandro Portes (2013) sobre el uso general del concepto de capital social permite comprender cómo dicho concepto ha ido evolucionando, desde su sentido más estricto de acceso a recursos a través de los vínculos que la persona establece con otras personas, o de aquellos que puede lograr por su pertenencia a distintas organizaciones, es decir, desde un enfoque micro donde la unidad de análisis es el individuo, hasta un uso generalizado y peligrosamente simplificado, a nivel macro, que se sostiene en in- 
dicadores como la confianza en el prójimo (un prójimo genérico), la confianza en las instituciones o la participación asociativa, visión estimulada por los trabajos de Putnam (por ejemplo, Putnam 2000).

En línea con la argumentación de Portes, muchos trabajos que han relacionado el capital social (concepto teórico que incentivó el avance del aparato teórico y metodológico del análisis de redes sociales) con las redes lo hacen desde esta posición centrada en el individuo, generalmente en la persona inmigrada. Desde el punto de vista de la estructura de sus redes personales, el capital social puede proceder de la cohesión social (bonding) que resulta de las conexiones intragrupo, de clausura, más horizontales y asociadas a la confianza, la identidad, la solidaridad y las normas, o de la integración social (bridging/linking) que surge de las conexiones inter-grupos, más abiertas y que se vinculan con la innovación, la información, la competitividad (Lozares et al. 2013).

La forma de indagar por el capital social ha variado, no obstante, según las fuentes. El estudio casi seminal de Aparicio y Tornos (2005) usa una encuesta propia sobre apoyo social dirigida a chinos, ecuatorianos, marroquíes, rumanos y senegaleses, y muestra diferencias por nacionalidades, entre otros aspectos por las atribuciones y obligaciones que se asocian con diferentes tipos de lazos, especialmente familiares. Otros autores han usado la Encuesta Nacional de Inmigrantes (ENI) para estudiar el acceso de los inmigrantes al empleo (Vono y Vidal-Coso, 2012) o el efecto de las redes sociales en las transiciones al matrimonio (del Rey y Vono, 2014).

En ocasiones, se pregunta directamente sobre la cantidad de contactos (parientes directos, parientes políticos, amigos) en el lugar de destino u origen. Así se mide, por ejemplo, en la encuesta EUMARR, con la que Koelet y Valk (2016) comparan las redes de inmigrantes parejas mixtas y endógamas en distintas ciudades europeas y su efecto sobre la soledad relacional.

Martínez García et al. (2001, 2002), por su parte, recogen datos primarios a partir de encuestas sobre apoyo social a inmigrantes africanos, y mujeres peruanas y marroquíes en distintas provincias andaluzas. Obtienen como indicadores el tamaño percibido de la red, la necesidad de apoyo, la utilización de la red y la satisfacción con el apoyo recibido.

\section{REDES SOCIOCÉNTRICAS Y ENCLAVES MULTI-ÉTNICOS}

El análisis de redes se inició con el estudio de las redes completas o sociocéntricas y, aunque muchas de las técnicas desarrolladas para ellas se han empleado también en el estudio de redes egocéntricas (en el siguiente apartado), en sentido riguroso sus supuestos de partida se ajustan a las primeras. Una red se define por un conjunto de actores y la relación que se establece entre ellos (Requena Santos 1989; Wasserman y Faust 2013). Supongamos que se quiere investigar sobre las relaciones inter-étnicas en un centro penitenciario. Si se analiza la red de confianza en un módulo concreto, los reclusos serían los actores y la relación entre ellos podría venir definida, por ejemplo, por la pregunta ¿en 
quién/es confías?, o ¿de quién/es sientes miedo? El contexto y la población de estudio deben quedar claramente acotados para que no existan dudas sobre quién pertenece y quién no a la red que se desea estudiar. Esta cuestión, en apariencia banal, en la práctica puede suponer una limitación a la que es difícil ajustarse. Si se analiza, por ejemplo, la red de ONGs de inmigrantes que operan en cierto ámbito geográfico y se pregunta ¿con qué ONGs has colaborado en alguna iniciativa común?, podría suceder que apareciera alguna no contemplada inicialmente en el conjunto. Se deben, por tanto, buscar criterios operativos que permitan recoger datos de todos los actores y que los vínculos que se dibujen se restrinjan a los incluidos en el grupo de estudio.

En cualquier caso, una vez delimitado el conjunto de nodos o actores (que pueden ser personas, organizaciones, países, etc.) y la relación que define la existencia o no de un lazo entre cada par de ellos, se procede a la recogida de datos. Lo más habitual es realizar este paso a través de la realización de una encuesta en la que cada actor (el preso, un representante de la ONG, etc.) responda por sí mismo o la entidad a la que representa ${ }^{2}$. Se pregunta entonces por los actores con quienes está relacionado. Esta relación puede ser dirigida, como en los ejemplos presentados en el párrafo anterior, o no dirigida (¿con quién has coincidido en algún taller o actividad desarrollada en la prisión?). En las dirigidas, las relaciones no tienen que ser necesariamente recíprocas, podría ocurrir que la persona en quien se confía no sienta la misma confianza a su vez por la otra y, por tanto, este nexo no sea mutuo, pero en las no dirigidas las respuestas de los dos actores implicados en una díada o par deben coincidir (si A afirma haber compartido un taller con B, B tendría que afirmar lo mismo sobre A).

Normalmente, al final del cuestionario (pero estas pueden ir también antes, dependiendo del tipo de administración de encuesta y de otras valoraciones metodológicas) se incluirán las preguntas que recopilen aquellas características de interés sobre los informantes, como su lugar de nacimiento, su nacionalidad, su edad, etc. (Requena Santos, 1996). La figura 1 representa dicho supuesto. Obsérvese que en el ejemplo se ha considerado una relación dirigida (B) y una relación dirigida valuada $(\mathrm{C})$, en la que el vector entre dos actores aparece ponderado (por ejemplo, si se indagara por el nivel de confianza: nada (sin lazo), algo o total). La forma, el color o el tamaño de los nodos pueden emplearse para reflejar sus características (D).

${ }^{2}$ Para este tipo de enfoques se pueden usar datos macro. Por ejemplo, se podrían analizar las redes de flujos migratorios entre provincias españolas (Abad Montes y Vargas Jiménez 2004) o entre países en el sistema mundial (Ruiz-Santacruz 2019). 
Figura 1. Recogida de datos de redes sociocéntricas

(A)
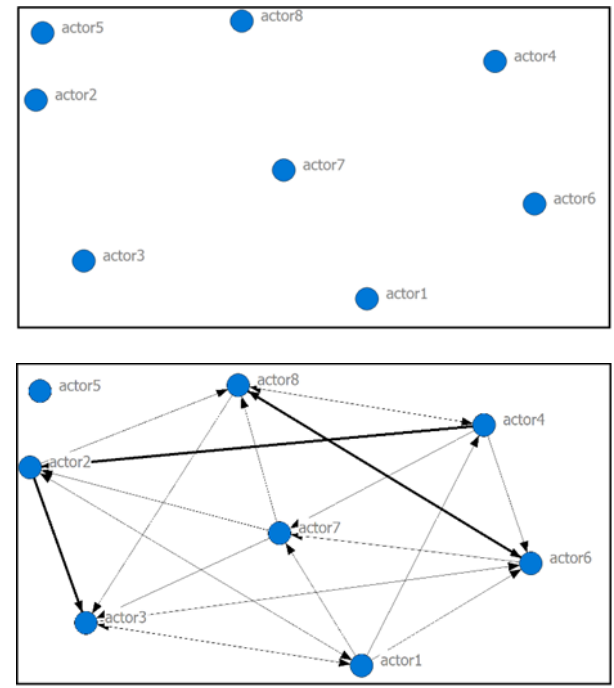

(C)
(B)
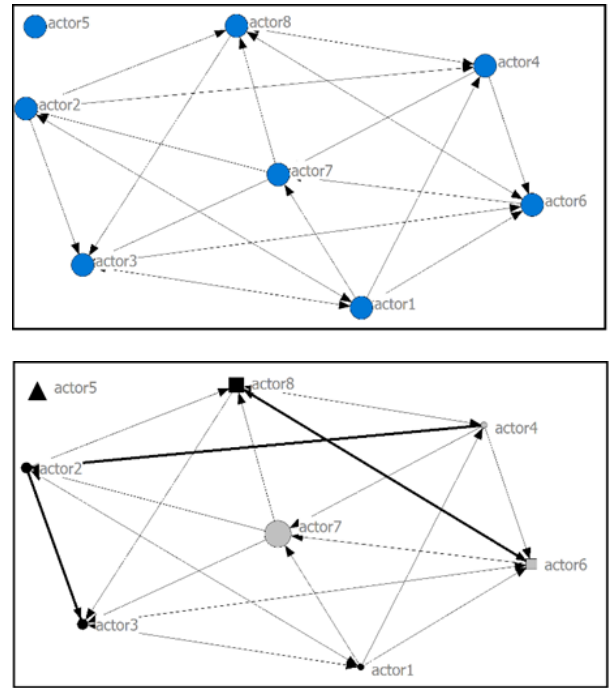

(D)

Fuente: elaboración propia

Tanto en este tipo de redes, como en las que se tratarán a continuación, se distinguen dos tipos de indicadores: los de composición, referidos a las características de los actores de la red, y los de estructura, que señalan propiedades de las conexiones que se establecen entre ellos (Requena Santos 1989; Wasserman y Faust 2013). Un ejemplo de los primeros sería el porcentaje de extranjeros en el módulo de la prisión, la edad media de los reclusos, etc. Entre los segundos, la densidad (cociente entre los lazos observados en la red y los posibles) es quizás el más usado e intuitivo, ya que indica hasta qué punto el entramado es tupido. Las implicaciones que tiene son obvias. Un grupo de clase en el que la relación estudiada sea la del intercambio de apoyo con las tareas, una densidad alta sería síntoma de la buena sintonía general del alumnado. Otros indicadores de la estructura son, por citar algunos, la identificación de subgrupos cohesivos (que pueden reflejar la existencia de sectores enfrentados), la presencia de tríadas transitivas, la localización de los actores más centrales en la red (la centralidad de grado, la de intermediación y la de cercanía...), que son los más populares o poderosos, en relaciones positivas, o los más conflictivos, en negativas ${ }^{3}$, etc.

${ }^{3}$ Para el éxito de programas de intervención, puede ser fundamental activarlos o acudir a ellos (Maya Jariego 2016). 
En la investigación en el contexto español este enfoque metodológico ha sido, con diferencia, bastante menos extendido. La literatura anglosajona nos ofrece múltiples ejemplos de enclaves multi-étnicos, entre los que el escolar es probablemente el más fecundo (Baerveldt et al. 2004; Lubbers y Snijders 2007). El estudio de las redes completas permite el acercamiento a la estructura de oportunidades. Si se controla las opciones reales que la persona tiene de establecer relación con otras en el contexto - acotado - estudiado, las posturas de acercamiento o rechazo se podrán valorar de forma más certera y en su justa medida. En una clase de 30 estudiantes donde diez de ellos son chinos, las probabilidades de establecer vínculos intra-grupo serán obviamente muy superiores a las del aula donde solo se hallan tres. Los modelos estadísticos desarrollados para el análisis de la probabilidad de que exista un vínculo o no (ERGM, por sus siglas en inglés: exponential random graph models, o $p^{*}$, que supuso la mejora del anterior modelo $p^{2}$, inspirado a su vez en el modelo logístico), tienen en cuenta esta estructura, así como también la tendencia a la homofilia o formar díadas con personas similares a uno mismo (el británico con el británico), o la inclinación natural en un gran número de relaciones a la transitividad (el amigo de mi amigo es mi amigo). Usar un simple modelo logístico para estimar la probabilidad de existencia o no de lazo entre dos actores supondría ignorar el supuesto de independencia de las unidades de observación, que sí tienen en cuenta los modelos específicos de análisis de redes, y dificultaría la incorporación de variables sobre estructura.

Este tipo de modelos, muy potentes, presentan el inconveniente de que pueden no converger y, por tanto, no llegar a ofrecer una solución óptima cuando ciertos atributos de los nodos están poco representados en la red, por ejemplo. Además, hay que tener presente que son modelos que se aplican a una red (RuizSantacruz 2019, para la red de emigraciones mundial). Si se quisiera estudiar todo un conjunto de redes, se tendría que recurrir a un meta-análisis, que es una aplicación particular del análisis multinivel (Baerveldt et al. 2004; Lubbers y Snijders 2007; de Miguel Luken y Carvajal Gutiérrez 2007). Por ejemplo, si la pregunta de investigación fuese si la relación de acoso escolar se da con mayor probabilidad entre estudiantes de distinto origen nacional y se realizara la recogida de datos reticulares en 20 grupos diferentes de clase, se tendría que elaborar un meta-análisis para poder extrapolar conclusiones generales sobre todos los grupos.

Es también para las redes sociocéntricas o completas para las que se han desarrollado los modelos longitudinales. ¿Cómo evolucionan los vínculos? ¿Cómo se desvanecen o se forman otros nuevos? ¿A qué causas responden? Son especialmente útiles en relación con la disyuntiva influencia o selección (fumo porque mis amigos fuman o me hago amigo de los que fuman porque yo fumo) o para el análisis de los procesos de contagio de actitudes, opiniones, enfermedades..., ya que permiten comprender las dinámicas y los cambios producidos en la estructura de la red, en función de las condiciones estructurales de partida, pero igualmente de su composición (Snijders 2005, Steglich y Snijders 2010) 
Si bien el programa StOCNET permitía la ejecución de los modelos de naturaleza transversal, ERGM o p*, hace ya algún tiempo que no se sigue manteniendo y su cálculo se ha incorporado a paquetes de $R$, como pnet o statnet. De la misma manera, SIENA, para el análisis longitudinal de las redes, que anteriormente operaba en StOCNET se adaptó a $R$ como RSiena (Ripley y Snijders 2018). Otros programas permiten análisis de redes, sobre todo en lo que se refiere a la visualización o cálculo de indicadores de estructura, incluso correlación entre redes, etc., como el propio Stata (Grund 2015) o Ucinet (Hanneman y Riddle 2005, Borgatti et al. 2013), este último de interfaz más amigable, pero la tendencia que se desprende de los últimos congresos en análisis de redes es la del uso cada vez más masivo y generalizado de $R^{4}$. Además, $R$ es sumamente flexible para la agregación de las nuevas opciones de análisis que continuamente se van generando y que complementan las ya conocidas del ARS, como es el caso del análisis reticular de coincidencias (Escobar y Tejero 2018).

\subsection{Ejes temáticos principales}

Los escasos estudios para el caso español inciden en las relaciones inter-grupos de acuerdo fundamentalmente con la variable lugar de nacimiento. Alegre Canosa $(2005,2008)$ trata la cuestión en centros educativos de secundaria con una recogida de datos basada en observación directa, que completa con entrevistas en profundidad. Para su análisis, elabora visualizaciones y calcula indicadores de estructura (centralidades de varios tipos y camarillas). Esta aproximación metodológica le permite llegar a la conclusión de que los propios adolescentes de origen inmigrante interpretan que están integrados relacionalmente cuando sus redes son heterogéneas e incorporan amigos y amigas autóctonos/as (Alegre 2005). Sus resultados no constituyen exactamente una prueba de la hipótesis del contacto, según la cual los prejuicios y el racismo suelen surgir de la ignorancia, y es el roce con el otro, el conocimiento mutuo, el que lima las diferencias, desdibuja las creencias reduccionistas y elimina las hostilidades (Allport 1954). Según el autor, el posicionamiento de partida, el mayor o menor grado de sensibilización previo, actúa como impulsor o constrictor del contacto. Además, entre los más jóvenes, puede darse el criterio de excepcionalidad, el vínculo con el extranjero es una excepción a la regla, es mi amigo/a aunque al colectivo se le siga juzgando de forma estereotipada.

Por su parte, de Miguel Luken y Carvajal Gutiérrez (2007), también para centros educativos y analizando la relación de amistad, tras una primera parte descriptiva basada en grafos ${ }^{5}$ e indicadores clave, realizan modelos ERGM de

${ }^{4}$ Hay otros programas específicos de análisis de redes, como Pajek, Gephi, NetMiner, ORA... Para una revisión, véase Huisman y Van Duijn 2011.

5 Aunque matemáticamente el concepto de grafo implica dos conjuntos, el de actores y el de vectores o líneas entre ellos, aquí (como se observa a menudo en otros textos sobre redes) se usará esporádicamente como sinónimo de visualización o gráfico de la red. 
doce grupos de $2^{\circ}$ de la ESO con importante presencia y variedad geográfica de alumnado de origen extranjero. Sus resultados apuntan a una falta de coherencia entre los discursos desprendidos de las preguntas generales sobre opiniones y actitudes ante la inmigración con respecto a los diferentes colectivos y las pautas relacionales que mantienen en la realidad del entorno educativo. De hecho, son los alumnos y alumnas marroquíes y británicos los que mejor expresan dicha contradicción, ya que no se revelan indicios claros de discriminación relacional hacia los marroquíes, latinoamericanos, asiáticos o resto de europeos y sí es significativo el relativo aislamiento de los británicos, que destacan por su tendencia a la homofilia por origen.

Para el ámbito español y en relación con el tema de las migraciones no hay, hasta donde han podido descubrir la autora de este texto, otras investigaciones que adopten la perspectiva de las redes sociocéntricas.

\section{Redes egocéntricas de la población inmigrada}

El enfoque de redes egocéntricas es el más empleado dentro del ARS para el estudio de la población inmigrada. La recogida de datos en este caso consiste en que el propio informante (ego), normalmente la persona inmigrada, enumera a las distintas personas que componen su red (y que se denominan alteri), a través de preguntas definidas como 'generadores de nombres', y posteriormente nos ofrece datos sobre las características de esas personas mencionadas ('intérpretes de nombres') (Requena Santos 1996). En el estudio de las migraciones, los generadores de nombres a menudo son generales y se inquiere simplemente por personas que se conozcan (por ejemplo: Lubbers et al. 2007; Ávila 2008; Bolíbar et al. 2013, 2015; Lubbers y Molina, 2013), y en ocasiones son más específicos y se vinculan con los tipos de apoyo intercambiado (de Miguel Luken et al. 2007; de Miguel-Luken y Tranmer 2010; Kornienko et al. 2018), o percibido (Cachia y Maya Jariego 2018). El lugar de nacimiento, la nacionalidad o el lugar de residencia suelen constituir variables de interés sobre los alteri por cuanto nos sugieren sobre la integración relacional con la población autóctona o la dispersión geográfica de las redes y su carácter transnacional. Finalmente, en una tercera fase, se pregunta al informante por los lazos existentes entre cada par de personas mencionadas ('matriz de relaciones'). La figura 2 ilustra un sencillo ejemplo hipotético, en el que ego menciona a 6 alteri (A), luego aporta información sobre el lugar de residencia de cada alter (color: España/extranjero), su lugar de nacimiento (forma: España/extranjero) y su proximidad emocional con ellos (tamaño según una gradación de 1 a 5, donde 1 es 'nada cercanos/relación conflictiva' y 5 'muy cercanos/relación íntima') (B), y finalmente indica si se conocen entre ellos o no (existe una línea que une a cada dos alteri o no) (C). Es esta última parte la que da lugar a la información puramente reticular, la que indica cómo es la estructura de la red. Por ello, normalmente se extrae a ego de la visualización y de la matriz de datos resultante (matriz de adyacencia) ya que, al estar unido a todos los alteri, su inclusión es información no relevante. 


\section{Figura 2. Recogida de datos de redes egocéntricas ${ }^{6}$}

(A) Generador de nombres

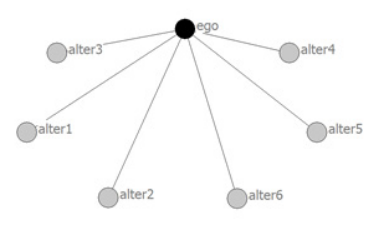

(B) Intérpretes de nombres

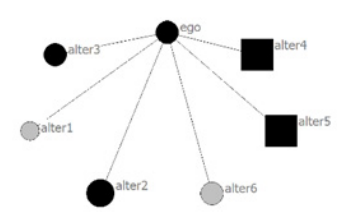

(C) Matriz de relaciones

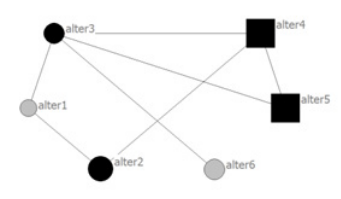

Fuente: elaboración propia

Con respecto a la cantidad de alteri y, por consiguiente, al tamaño de las redes egocéntricas que se obtengan, esta dependerá de los generadores de nombres escogidos en función de la pregunta de investigación que se desea responder. La perspectiva puede ser abierta ( $n$ personas que conozcas $)^{7}$, aunque luego se inquiera por el apoyo intercambiado con cada una, o más concreta, cuando los generadores se plantean directamente en torno al apoyo (de Miguel Luken et al. 2007; de Miguel-Luken y Tranmer 2010; Herz 2015; Cachia y Maya Jariego 2018; Durán Durán y Arenas García 2018), o a la red emocional más íntima, siguiendo el modelo de la General Social Survey americana (Burt 1984; Viry 2012; Popielarz y Cserpes 2018), dando lugar a redes más pequeñas. Aunque McCarty (2002) argumenta que 30 es un tamaño de red personal lo suficientemente grande como para poder realizar un análisis estructural de la red y este límite ha sido aplicado con frecuencia (Bolíbar et al. 2013, 2015; de Miguel Luken et al. 2015; Bolíbar 2016, 2019; Hosnedlová 2017), otros estudios para España han ampliado a 45 el número de personas conocidas (Ávila 2008; Lubbers et al. 2007; Lubbers y Molina 2013, Vacca et al. 2018) o reducido la cantidad a 25 (Maya-Jariego y Armitage 2007; Repke-Benet-Martínez 2018). En estos casos, el generador debe ser lo suficientemente general y abierto para que todas las personas entrevistadas puedan llegar a mencionar ese número de conocidos. Un recurso ampliamente empleado es el de solicitar los nombres de personas con las que se haya tenido algún contacto en los dos últimos años por cualquier vía (cara a cara, por internet, etc.) y a las que se pudiera contactar de nuevo si se quisiera (Lubbers et al. 2007, Bolíbar et al. 2015). En realidad, se insiste en que ego dé el nombre, iniciales, apelativo, etc., de la forma que desee (asegurándole la posterior anonimización de dichas menciones), de manera que luego recuerde de quién está hablando en los intérpretes de nombres y la matriz

${ }^{6}$ Los programas más usados a este efecto son Egonet (https://sourceforge.net/projects/egonet/) y Vennmaker (http://www.vennmaker.com/?lang=en).

${ }^{7}$ Aunque aquí y en otros trabajos se use indistintamente el término de red egocéntrica y red personal, otros autores prefieren reservar el uso de red personal a las de este tipo (Lubbers y Molina 2013) 
de relaciones. El problema que puede tener este tipo de generadores es que los lazos fuertes se vean priorizados, si bien a partir de 25 alteri ya se recoge la suficiente cantidad como para que aparezca una buena representación de lazos débiles.

Por lo que se refiere a la matriz de relaciones, hay que evitar en lo posible los problemas de fiabilidad en las respuestas derivados de preguntas que sean difíciles de contestar para ego. Por ejemplo, de Miguel Luken et al. (2007) plantearon a los entrevistados tres tipos de relaciones entre los alteri: A) si había habido intercambio de apoyo entre ellos, B) si eran parientes, C) si había entre ellos una relación estrecha. En el trabajo de campo, se comprobó que muchos dudaban en la respuesta a la primera relación, por lo que se tuvo que prescindir de ella en la posterior explotación de los datos. Es por ello que, cuando se indaga por redes personales de mayor tamaño, normalmente el vínculo se define de una forma mucho más sencilla: si los dos alteri se conocen entre ellos o no, o si contactarían entre ellos con independencia de ego. Maya-Jariego y Armitage (2007) propusieron valuar el lazo entre cada par de alteri de 0 ("no hay relación") a 2 ("muy relacionados"), si bien lo habitual, por simplicidad y porque hay ciertos indicadores que no se pueden calcular para relaciones valuadas, es dicotomizar posteriormente la relación.

A menudo, cuando se aplican métodos mixtos, se ofrece a la persona entrevistada una visualización de la red personal que ha ido configurando a lo largo de la cumplimentación del cuestionario de redes. Así, una vez que esta parte más estructurada de la entrevista ha finalizado, el entrevistador se sirve de la entrevista en profundidad para detectar posibles inconsistencias o fallos en la recogida de datos reticulares, para completar la entrevista a la luz de los resultados, lo que suele facilitar el discurso del informante (Lubbers et al. 2007, Lubbers y Molina 2013, Hosnedlová 2017), y le conduce a profundizar mucho más en la narrativa relacional a lo largo de su biografía (Bolíbar et al. 2013, 2015; Bolíbar 2016, 2019; Verd et al. 2017). En ocasiones, es la propia entrevista cualitativa, complementada con mapas de redes y generadores de nombres, la que sirve para recoger los datos reticulares de una forma más espontánea (Bojarczuk y Mühlau 2018)

En cuanto a las técnicas y modelos estadísticos que se suelen aplicar en el análisis de las redes personales, una gran cantidad de aportaciones se centra en la descripción de las redes, recurriendo a la visualización de las mismas o a resúmenes de las características y composición en función de determinados colectivos. Como formas más habituales de presentación de resultados de esta naturaleza se recurre a tablas de contingencia, ANOVA o test de diferencias de medias, así como a la creación de tipologías (a menudo a través del análisis de conglomerados), en las que encajan los diferentes grupos nacionales. En muchas aportaciones su carácter es meramente descriptivo y constituye el objetivo de parte de la investigación que se presenta (Maya 2002, Bolíbar et al. 2015), aunque las tipologías también cumplen su función como variable explicativa (Lubbers et al. 2007, Cruz Gómez 2013, Vacca et al. 2018). Algunas propuestas solo incluyen variables de composición (Federico de la Rúa 2004, Bolíbar et al. 2013, 2015; Bolívar 2019) y otras también tienen en cuenta indicadores de 
estructura (Ávila Moreno 2008, Cachia y Maya 2018, Vacca et al. 2018). En general, la mayoría consideran el lugar de residencia y de nacimiento de los alteri y contrastan la parte local con la transnacional, la presencia de autóctonos frente a la de connacionales.

Cuando el número de alteri por los que se pregunta es elevado y se quiere comparar un número de redes considerable, se recurre a veces a la simplificación de la misma a través de la síntesis de toda la información relacional y de composición con respecto a una o varias características particulares de los alteri, en las que los investigadores están especialmente interesados (figura 3). Se trata de los grafos de conglomerados (clustered graphs) (Brandes et al. 2008; Lubbers et al. 2010; Bolíbar et al. 2015; de Miguel Luken et al. 2015; Verd et al. 2017; RepkeBenet-Martínez 2018) o metarrepresentaciones (Molina et al. 2008).

Figura 3. Ejemplo de reducción de la información de la red personal en un grafo de conglomerados, a través del programa Visone

RED ORIGINAL

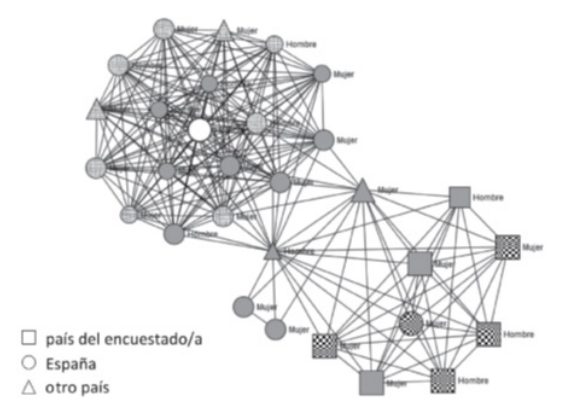

\section{METARREPRESENTACIÓN}
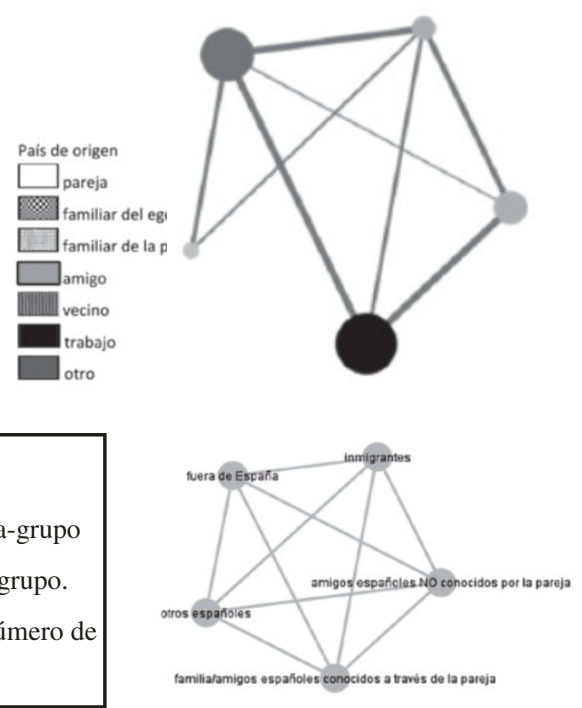

Leyenda de la metarrepresentación

Color del nodo: densidad de las relaciones intra-grupo

Tamaño del nodo: número de actores en el subgrupo.

Grosor del segmento que une los subgrupos: número de interrelaciones

Fuente: adaptado de Miguel Luken et al. 2015: 161, 162, 168

En cuanto a los modelos explicativos, lo idóneo es el análisis multinivel cuando la variable dependiente es una característica del alter, o de la díada alterego. Es fácil observar en la figura 2 la estructura anidada de los datos (A). Los alteri están anidados en cada ego, por lo que no sería lógico presumir a priori la independencia de las observaciones al segundo nivel, o nivel de alter. Esta 
técnica evita los errores derivados del cálculo de estimadores sesgados y de la subestimación de los errores estándar que resultarían de ignorar dicha estructura de los datos (van Duijn et al. 1999). Como ejemplo, en términos de integración relacional podría interesar si las personas mencionadas son autóctonas o no (de Miguel Luken y Tranmer 2010), o qué tipo de apoyo han aportado (Herz 2015, Kornienko et al. 2018). Esta misma técnica debería aplicarse en el estudio longitudinal de las redes personales, si lo que se pretende por ejemplo es cuestionar la perdurabilidad o no de los lazos ego-alter (Lubbers et al. 2010, Lubbers y Molina 2013).

En cambio, si el objetivo radica en analizar la existencia o no de lazos entre los propios alteri, o la evolución de los mismos, se podría considerar que la estructura de dependencia es similar a la de las redes sociocéntricas y usar modelos similares a los aplicados para las mismas (Lubbers et al. 2010), los ya comentados ERGM o SIENA.

En ocasiones, las variables referidas a composición y estructura actúan como predictores de otro tipo de características no relacionadas con la red, sino con ego. Variables como la cohesión de ciertos subgrupos, el tamaño de la red, la densidad, el porcentaje de autóctonos o el tipo de red de acuerdo con alguna tipología actúan en este supuesto como variables independientes. Este tipo de preguntas de investigación a nivel de ego se responden aplicando técnicas multivariantes convencionales. Para el contexto español, se encuentran ejemplos en modelos para estimar la escala de aculturación (Vacca et al. 2018), el nivel de bienestar de la población inmigrante o la continuidad de las cadenas migratorias (de Miguel Luken et al. 2007), el tipo de identificación social (Lubbers et al. 2007), o la intención de permanecer en España (Hosnedlová 2017).

\subsection{Ejes temáticos principales}

Es sumamente complicado realizar una división exhaustiva y excluyente de áreas temáticas en el estudio de las migraciones desde el ARS. Los grandes temas de interés se solapan en las investigaciones y no existen líneas divisorias nítidas. No obstante, se propone la siguiente distinción operativa: integración relacional o social (el foco de atención se centra en la creación de ligámenes con la población autóctona), apoyo social y sus tipos, y evolución de las redes. En estas temáticas es común que aparezca (cada día más) como eje transversal el del transnacionalismo.

a. Integración relacional

El acento se ha puesto en general en la participación de la población autóctona en las redes de las personas inmigradas y en las diferencias halladas por nacionalidad (Maya 2002, de Miguel Luken et al. 2007, 2015; de Miguel Luken y Tranmer 2010; Lubbers y Molina 2013; Verd et al. 2017), aunque también la perspectiva de género en la composición y estructura de las redes personales ha sido recurrente en el estudio de las redes personales y de apoyo (Bolívar 2016). 
Los colectivos marroquí y ecuatoriano probablemente hayan sido de los más analizados $\mathrm{y}$, aunque muchos estudios apuntan la menor presencia de españoles en las redes de los marroquíes con respecto a otras nacionalidades (Martínez García et al. 2001, de Miguel Luken et al. 2007; de Miguel Luken y Tranmer 2010; Lubbers y Molina, 2013), otros contradicen esta conclusión (Repke y Benet-Martínez 2018).

La formación de parejas mixtas, formadas por un miembro autóctono y el otro de origen extranjero, ha sido tradicionalmente aceptada como un síntoma, por lo menos a nivel agregado, de la asimilación de la población inmigrante (del Rey y Vono 2014, Koelet y De Valk 2016). De todas formas, esta afirmación ha recibido abundantes réplicas y algunos autores llegan a señalar que se producen procesos de "integración a la inversa", en los que son los nativos los que se insertan en los círculos sociales de sus parejas extranjeras (Rodríguez García et al. 2014). El estudio de la composición de las redes de inmigrantes en uniones mixtas constituye una estrategia óptima para descubrir si, en efecto, a escala micro estas personas inmigradas con pareja autóctona son más proclives a contar con españoles en sus redes personales, si lo son aun obviando a los vínculos heredados por la familia política, o si la propia formación de vínculos inter-étnicos entre alteri son más probables en su círculo social que en el de personas en unión endógama (de Miguel Luken et al. 2015).

De alguna manera, la identificación con la comunidad receptora se puede considerar una consecuencia de la integración social (Gualda 2008). El apoyo recibido de unos grupos étnicos u otros y el número de amigos y contactos de diferentes nacionalidades potencian el sentimiento de identidad con comunidades en unos u otros límites espaciales (Federico de la Rúa 2004, García Faroldi 2008, Gualda 2008). En la dirección opuesta, el número de compatriotas en la red personal y en la red de apoyo correlaciona positivamente con un sentido de comunidad más ligado al origen (Maya-Jariego y Armitage 2007), o una integración bicultural (Repke y Benet-Martínez 2018). Además, características estructurales de la red personal y la posición que en ella ocupan determinados actores con un liderazgo destacado ayudan a propagar en la red la identificación con un determinado lugar y pueblo, generalmente ensanchando los límites geográficos de la identificación (García Faroldi 2008). Las redes más densas se asocian con un mayor consenso en las identificaciones de sus miembros y las redes con múltiples subgrupos tienden menos claramente a identificaciones de tipo étnico (Lubbers et al. 2007). Además, la presencia física no es óbice para sentirse identificado con un lugar o colectivo, ni las identificaciones están necesariamente limitadas a ser únicas, pudiendo existir múltiples identificaciones (Gualda 2008; Repke y Benet-Martínez 2018).

Dentro de la integración social, la investigación sobre el asociacionismo inmigrante se ha preocupado por descubrir si la participación en este sentido ha incitado a la socialización con personas autóctonas (teoría de la integración) o, por el contrario, cuando las asociaciones se han caracterizado por la homogeneidad de sus miembros, ha propiciado el encapsulamiento o segregación de ciertas 
comunidades (teoría del enclave étnico) (Bolíbar 2011, 2019; Bolíbar et al. 2013; Popielarz y Cserpes 2018).

\section{b. Apoyo social}

Otro bloque de estudios hace hincapié en la provisión específica de apoyo. En ellos, no se trata tanto de conocer la composición de la red personal, como de dibujar el entramado activado o activable en la provisión de ayuda, ya sea de tipo emocional como instrumental. Algunos autores muestran que el tamaño importa (Martínez García et al. 2001, 2002; Hosnedlová 2017), y que las redes que peor satisfacen las necesidades de la población inmigrada son las más pequeñas. Las redes de apoyo en el destino, al menos en una primera fase tras la inmigración, oscilan entre los 4 y los 11 vínculos en promedio, siendo más reducidas que la de los nativos, lo que repercute en una mayor multiplicidad de los vínculos, que asumen un rol polifacético en su provisión de apoyo (Maya Jariego 2006). Asimismo, algunas aportaciones señalan el mayor protagonismo de los autóctonos en la provisión de información (Martínez García et al. 2001) y, en cambio, otros establecen su relevancia en la asistencia material y económica (de Miguel Luken et Tranmer 2010). En cualquier caso, hay que tener en cuenta que los estudios citados hacen referencia a distintos momentos de tiempo, con diferentes niveles absolutos y relativos de presencia de inmigrantes en España. En estos trabajos no se puede controlar la estructura de oportunidades.

La provisión de apoyo no se hace únicamente necesaria en una primera fase de adaptación en el nuevo país de residencia, aunque pueda tratarse del periodo en el que más se activan estos vínculos. Las diferentes etapas del ciclo de vida llevan asociadas diferentes necesidades que, dependiendo de las opciones que los mecanismos formales de apoyo brinden en el país, van a ser relegadas a los lazos fuertes o débiles disponibles. Es de esperar, además, que el tipo de apoyo determine en parte de quién proceda. Si la ayuda requerida es costosa en términos de tiempo o esfuerzo, procederá con más frecuencia de lazos fuertes, definidos estos como aquellos más próximos emocionalmente o con los que hay una mayor frecuencia de contacto (Granovetter 1973). Además, salvo en el caso de los familiares, la reciprocidad es un valor esperado del apoyo social (Bojarczuk y Mühlau 2018), aunque los lazos mutuos se dan más entre connacionales y se observan casi invariablemente en la ayuda emocional (Kornienko et al. 2018). Dicha ayuda emocional, junto con la económica, son las menos sensibles a la distancia geográfica (Molina et al. 2011, Viry 2012, Bolíbar et al. 2013, Herz 2015, Cachia y Maya 2018). Si la ayuda es instrumental y requiere de la presencia física del donante, como en el caso del cuidado de familiares enfermos, hijos, etc., necesariamente requerirá a los vínculos locales, establecidos cerca de la persona demandante (Herz 2015, Bojarczuk y Mühlau 2018).

c. Evolución de las redes

Hay escasas investigaciones en España que analicen la evolución de las redes desde una perspectiva puramente longitudinal, es decir, siguiendo a la población a lo largo del tiempo e indagando por sus redes personales en los distintos 
momentos de medición del fenómeno (Lubbers et al. 2010, Lubbers y Molina 2013). En cambio, por cuestiones obvias, es más común encontrar trabajos que se centran en la evolución de las redes a partir de la interpretación del efecto de la variable tiempo de residencia (Bolíbar et al. 2015), del momento en el que se conoció a cada alter (Bolíbar et al. 2015; Bolíbar 2016; Verd et al. 2017), o por la introducción de preguntas con carácter retrospectivo (de Miguel Luken et al. 2007).

La evolución más esperada de las redes es la correspondiente a la visión del asimilacionismo lineal, por el cual, con el paso del tiempo, estas incorporan cada vez más contactos originarios del lugar de destino en detrimento de los que residen en el lugar de origen (Molina 2005; Bolíbar et al. 2015). En la actualidad, la asimilación estructural coexiste con un transnacionalismo estructural, que Vacca et al. (2018) definen como el mantenimiento de las redes sociales con el grupo primario en la sociedad de partida. Las redes se destruyen y reconstruyen con la emigración (Maya Jariego 2006) y es previsible que, cuanto más prolongada la estancia en el destino, más se debiliten los nexos con el lugar de origen y se formen nuevos en el lugar de destino. Las redes comienzan siendo más cambiantes sobre todo en la primera fase en el destino, tras la emigración, produciéndose una elevada sustitución de los contactos conocidos en los primeros años tras la emigración (Maya Jariego 2006, Lubbers y Molina 2013, Bolíbar et al. 2015). Ávila (2008) encuentra que solo un $7 \%$ de las redes que analiza permanecen bastante estables en los dos años de lapso considerado entre sus mediciones y Lubbers y Molina (2013) sitúan en un 20\% la sustitución real de las redes en un periodo de dos años. En cualquier caso, a partir de un cierto momento, no parece existir una clara relación lineal entre el tiempo de residencia en España y la incorporación de autóctonos en las redes de los inmigrantes (Lubbers y Molina 2013, Bolíbar et al. 2015, Hosnedlová 2017). Verd et al. (2017) sostienen, en este sentido, que los tres primeros años de residencia en España son determinantes.

Sin embargo, este asimilacionismo relacional aparece a menudo cuestionado. En el desarrollo de las redes son múltiples los aspectos que intervienen, pero entre ellos cobran trascendencia especial los planes de reagrupación, el proyecto de futuro, ya que la perspectiva de permanecer en el destino predispondrá a la creación y mantenimiento de nuevos contactos allí (Bolíbar et al. 2015; Bolíbar 2016; Verd et al. 2017; Cacha y Maya 2018), el contexto de partida (la emigración es a veces planteada como huida), así como el de recepción (más o menos favorable) (Portes y Böröcz 1989), de tal manera que, en lugar de observarse una progresiva incorporación de autóctonos en las redes, se llegan a producir incluso procesos de involución (Lubbers et al. 2010) o de encapsulamiento en enclaves étnicos.

En la figura 4 se muestra un esquema- resumen de las contribuciones presentadas en ese espacio de intersección entre las redes sociales y las migraciones. Se han considerado diferentes ejes de acuerdo con la perspectiva metodológica empleada y los grandes ámbitos temáticos anteriormente comentados. Las categorías son difícilmente excluyentes, sobre todo en lo tocante a los temas de in- 
vestigación, pero se ha intentado posicionar las referencias donde mejor pudieran quedar clasificadas (algunas aparecen entre columnas porque abordan ambas temáticas). Prácticamente todos los trabajos que incluyen aspectos de la estructura de la red también lo hacen sobre su composición, por ello se han ubicado en la primera categoría. La distinción entre variable dependiente / variable independiente hace referencia a la función de las variables que miden algún aspecto de las redes sociales de la población inmigrante, cuando se incorporan en modelos multivariantes. Si la asociación entre variable dependiente e independiente se reduce a gráficos, tablas de contingencia o similar, se sitúa la referencia más cerca de la categoría 'descriptivo'. 


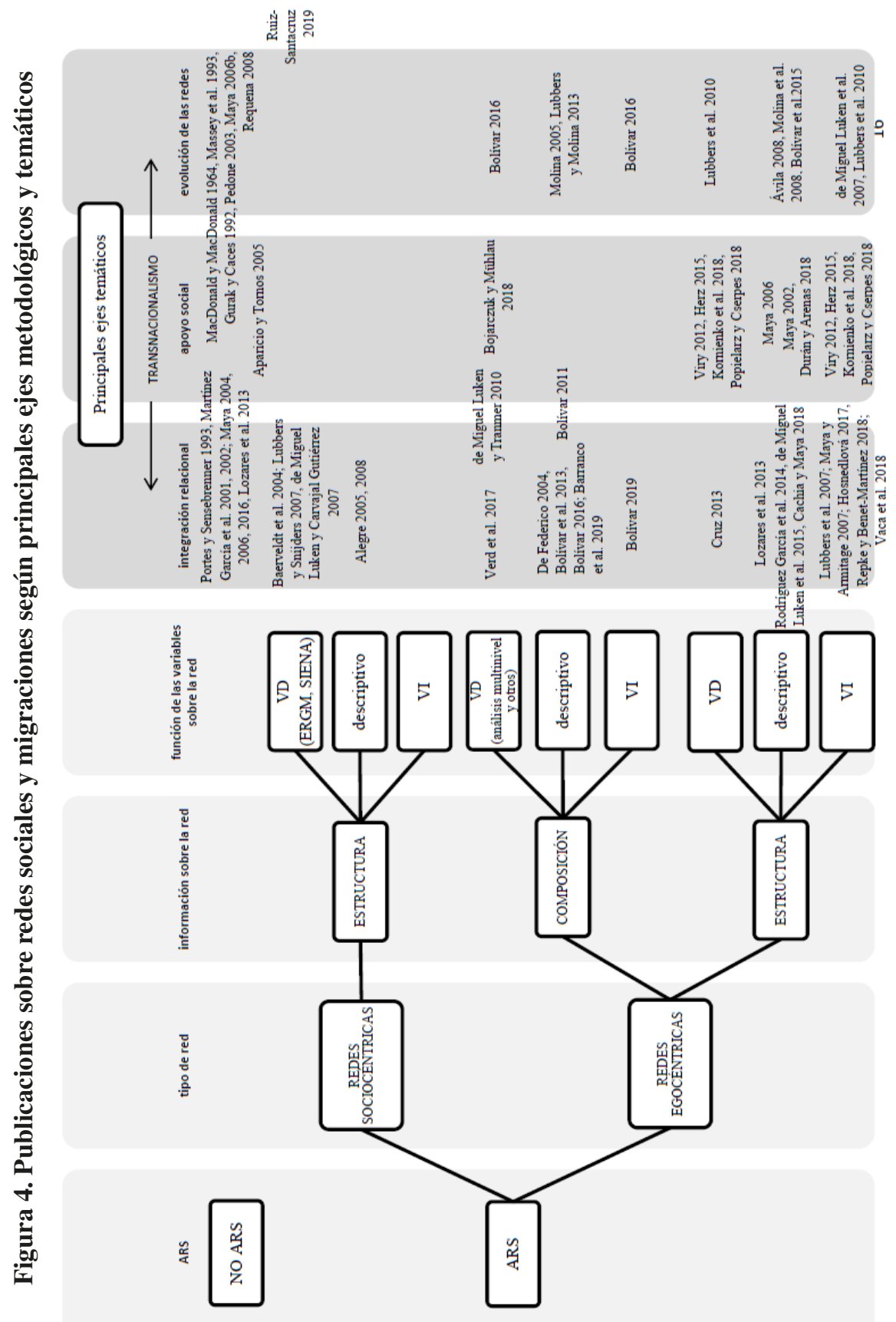




\section{A MODO DE CONCLUSIÓN: FORTALEZAS Y LIMITACIONES DEL ARS EN EL ESTUDIO DE LAS MIGRACIONES}

La inserción de la población extranjera no puede ser comprendida de la misma manera si se tiene en cuenta la estructura reticular del entorno inmediato en el que se desarrolla la vida de la persona inmigrante o no. No es lo mismo estar rodeado de un nutrido grupo de connacionales que pertenecen a diferentes submundos, que estar rodeado de un nutrido grupo de connacionales que se conocen entre sí. En el primer caso, la persona dispondrá de salidas a más realidades diferentes, acceso a información menos redundante. En el segundo, la presión y el control del colectivo serán mayores, pero también la confianza y la solidaridad que se establezca dentro del grupo. Tampoco tiene las mismas implicaciones conocer a un grupo de autóctonos que son familiares de la pareja de ego, que conocer a autóctonos de diferentes ambientes y espacios de vida. En las redes sociocéntricas, ser amigo de un líder en el grupo podrá conllevar un acceso más directo y rápido a los recursos. En el plano de la intervención, identificar los subgrupos cohesivos, los puentes entre ellos, las personas más carismáticas, repercutirá en el mayor éxito de las iniciativas. Controlar la estructura de oportunidades en el intercambio de consejo y ayuda profesional entre trabajadores de distintas procedencias de una empresa conducirá a conclusiones mejor fundamentadas sobre las relaciones inter-étnicas en dicho contexto. El conocimiento de la estructura de la red completa la perspectiva sobre su composición. Esa estructura incide sobre la acción individual, y la acción individual modifica a su vez la estructura.

La potencialidad del ARS al estudio de las migraciones se ha evidenciado a lo largo de este texto a través de los ejemplos mencionados. Las palabras de Vacca et al. (2018: 73) recogen muy claramente hasta qué punto el ARS permite ampliar la visión sobre los procesos de movilidad y de integración de la población que ofrecen otras aproximaciones metodológicas en las que está ausente la información reticular:

We argue that personal network structure contributes to a better understanding of assimilation and transnationalism patterns among international immigrants. A central intuition in sociology and social network analysis is that a group is more than the sum of its members, and a network is more than the sum of its dyadic ties. A cohesive group of personal contacts exposes the individual to a fundamentally different set of constraints, resources and opportunities than a set of five separate contacts.

Las limitaciones del análisis de redes son varias. Desde el punto de vista de las redes egocéntricas, las encuestas que recogen información sobre estructura y composición de las redes, además de las preguntas pertinentes sobre los egos, son costosas, largas, y tediosas en la parte reticular y se corre más fácilmente el riesgo de agotar a los informantes. La visualización de la red propia puede servir, no obstante, como aliciente que compense este exceso de esfuerzo. Por otra parte, aunque se pueda extraer una muestra más o menos representativa de la población de estudio (con las dificultades ya intrínsecas a cualquier investi- 
gación sobre población inmigrada), se debe tener precaución en evitar cualquier posible solapamiento entre los alteri de las redes personales de los informantes, que haría peligrar algunos supuestos estadísticos.

Desde el punto de vista de las redes sociocéntricas, una red es tan solo una pequeña porción de realidad que, a veces, además, es difícil de acotar. Obtener una muestra representativa de redes completas es también una empresa muy costosa, y las opciones de generalización de los resultados suelen ser reducidas, a pesar de la posibilidad de la realización de meta-análisis. Además, la complejidad de los modelos estadísticos del ARS puede conducir al investigador a prescindir de algunas de sus redes por problemas de convergencia en el desarrollo de los cálculos.

No se ha discutido en el texto sobre cuestiones éticas relacionadas con esta metodología y, sin embargo, es amplia la preocupación que despiertan. En las redes egocéntricas, ego proporciona datos de terceros que no han dado su consentimiento. En las sociocéntricas, la anonimización de los datos recogidos no es siempre efectiva, sobre todo en grupos pequeños, lo que puede plantear serios problemas a la hora de difundir los resultados.

Por otra parte, el tratamiento de los valores perdidos es un reto en esta metodología. ¿Qué se hace con la información del alumno que ese día no pudo ir a clase? ¿Y del actor que se pierde en un estudio longitudinal? ¿Qué criterio se adopta con los lazos omitidos? Estas y otras cuestiones son parte de un debate metodológico para el que todavía no hay respuestas totalmente satisfactorias.

Hay muchas vías para seguir avanzando y explorando en la cuestión. Aparte de las aportaciones técnicas e informáticas que no cesan y siguen siendo necesarias en un ámbito metodológico relativamente tan joven, faltan en nuestro contexto más próximo investigaciones sobre redes completas: en el ámbito laboral, en los centros educativos, en las prisiones, en las asociaciones y los clubs... e investigaciones que acometan el estudio longitudinal de las redes. Investigaciones que nos pueden hacer entender los procesos de selección e influencia, el dinamismo de las redes y las verdaderas relaciones causa-efecto.

\section{BIBLIOGRAFÍA}

ABAD MONTES, F. y M. VARGAS JIMÉNEZ (2004): "Una aproximación Social Network al estudio de la movilidad social: Análisis de una tabla de migración", IX Congreso de Población Española, Granada.

ALEGRE I CANOSA, M. À. (2005): Geografies adolescents a secundària. Els posicionaments dels fills i filles de famílies d'origen immigrant en els mapes relacionals i culturals articulats en l'àmbit escolar, Dep. de Sociología, Barcelona, Universidad Autónoma de Barcelona.

ALEGRE CANOSA, M. À. (2008): «Geografías adolescentes y contacto intercultural. Una visión desde el ámbito escolar», Papers. Revista de Sociologia, 90, pp. 33-58.

ALLPORT, G. (1954): The Nature of Prejudice, Cambridge, Addison-Wesley.

APARICIO, R. y A. TORNOS (2005): Las redes sociales de los inmigrantes extranjeros en España, Madrid, Ministerio de Trabajo y Asuntos Sociales. 
ÁVILA MOLERO, J. (2008): "Redes personales de africanos y latinoamericanos en Cataluña, España. Análisis reticular de integración y cambio", REDES- Revista hispana para el análisis de redes sociales, 15(5).

BAERVELDT, C., M. A. J. VAN DUIJN, et al. (2004): "Ethnic boundaries and personal choice. Assessing the influence of individual inclinations to choose intra-ethnic relationships on pupils' networks", Social Networks, 26, pp. 55-74.

BARRANCO, O., C. LOZARES, C. y D. MUNTANYOLA-SAURA (2019): "Heterophily in social groups formation: a social network analysis", Quality \& Quantity, 53(2), pp. 599-619.

BILECEN, B., M. GAMPER y M. J. LUBBERS (2018): “The missing link: Social network analysis in migration and transnationalism", Social Networks, 53, pp. 1-3.

BOJARCZUK, S. y P. MÜHLAU (2018): "Mobilising social network support for childcare: The case of Polish migrant mothers in Dublin", Social Networks 53, pp. 101110. doi.org/10.1016/j.socnet.2017.04.004

BOLÍBAR, M. (2011): "Las asociaciones en las redes personales. ¿Mecanismo de integración de la población inmigrante?", REDES- Revista hispana para el análisis de redes sociales, $20(7)$.

BOLÍBAR, M. (2016): "Macro, meso, micro: broadening the 'social' of social network analysis with a mixed methods approach”, Quality \& Quantity, 50, pp. 2217-2236. DOI 10.1007/s11135-015-0259-0.

BOLÍBAR, M. (2019): "Personal Networks and Participation: Relational Mechanisms for the Local and Transnational Civic Involvement of Immigrants", The Sociological Quarterly, 60(4), pp. 583-606.

BOLIBAR, M., J. MARTÍ y C. LOZARES (2013): “Aplicaciones de los métodos mixtos al análisis de las redes personales de la población inmigrada”, EMPIRIA. Revista de Metodología de Ciencias Sociales, 26, pp. 89-116. DOI: 10.5944/empiria.26.7154

BOLÍBAR, M., J. MARTÍ, y J. M. VERD (2015): "Just a question of time? The composition and evolution of immigrants' personal networks in Catalonia", International Sociology, 30(6), pp. 579-598. DOI: 10.1177/0268580915579925.

BORGATTI, S. P., M. P. EVERETT, et al. (2013): Analyzing Social Networks, London, SAGE.

BRANDES, U., J. LERNER, M. J. LUBBERS, J.L. MOLINA y C. MCCARTY (2008): "Visual statistics for collections of clustered graphs", Proceedings of the 2008 IEEE Pacific Visualization Symposium, Kyoto, Japan.

BURT, R. S. (1984): "Network Items and the General Social Survey", Social Networks, 6, pp. 293-339.

CACHIA, R. e I. MAYA JARIEGO (2018): "Mobility types, transnational ties and personal networks in four highly skilled immigrant communities in Seville (Spain)", Social Networks, 53, pp. 111-124.

CRUZ GÓMEZ, I. (2013): La homofilia como forma de estructuración de las relaciones sociales en Cataluña, Departamento de Sociología, Barcelona, Universitat Autònoma de Barcelona.

DURÁN DURÁN, A. y L. ARENAS GARCÍA (2018): "Social network analysis: emotional and instrumental links of women inmates", 18th Annual Conference of the European Society of Criminology, Sarajevo, Bosnia Herzegovina.

ESCOBAR MERCADO, R. M. y C. TEJERO (2018): "El análisis reticular de coincidencias”, EMPIRIA. Revista de Metodología de Ciencias Sociales, 39, pp. 103-128. 
FEDERICO DE LA RÚA, A. de (2004): “Los espacios de transnacionalidad. Una tipología de la integración relacional de los migrantes", REDES. Revista hispana para el análisis de redes sociales, 7(4).

GAETE FISCELLA, J. M. (2014): Redes interorganizativas y capital social: una aproximación estructural a la integración social de las asociaciones de inmigrantes, Departamento de Sociología y Comunicación, Salamanca, Universidad de Salamanca, 381 pp.

GARCÍA FAROLDI, L. (2008): Construyendo Europa. Las redes sociales en la difusión de actitudes e identificaciones hacia la Unión Europea, Madrid, CIS.

GRANOVETTER, M. (1973): The Strength of Weak Ties, American Journal of Sociology, 78(6), pp. 1360-1380.

GRUND, Thomas U. (2015) "nwcommands. Network Analysis in Stata". Recuperado de: http://nwcommands.org, el 25/10/18.

GUALDA CABALLERO, E. (2008): "Identidades, autoidentificaciones territoriales y redes sociales de adolescentes y jóvenes inmigrantes", Portularia, 8(1), pp. 111-129.

GUALDA, E. y REBOLLO DÍAZ, C. (2016): "The refugee crisis on twitter: a diversity of discourses at a European crossroads". Journal of Spatial and Organizational Dynamics, 4(3), pp. 199-212, (2016). ISSN 2183-1912. En http://rabida.uhu.es/dspace/ handle/10272/13624.

GURAK, D. T. y F. CACES (1992): "Migration networks and the shaping of international migration systems”. En: KRITZ, M. M., LIM, L. L. y ZLOTNIK, H. (eds.) International Migration Systems: A Global Approach. Oxford: Clarendon Press, pp. 150-176.

HANNEMAN, R. y M. RIDDLE (2005): Introduction to social network methods. En: http://www.faculty.ucr.edu/ hanneman/nettext

HERZ, A. (2015): "Relational constitution of social support in migrants' transnational personal communities", Social Networks, 40, pp. 64-74. dx.doi.org/10.1016/j.socnet.2014.08.001.

HOSNEDLOVÁ, R. (2017): "Embedded settlement intentions: The case of Ukrainians in Madrid", Social Networks, 49, pp. 48-66.

HUISMAN, M. y M. A. J. VAN DUIJN (2011): “A Reader's Guide to SNA Software". En: J. SCOTT y P. J. CARRINGTON, The SAGE Handbook of Social Network Analysis, London, SAGE, pp. 578-600.

KOELET, S. y H. A. DE VALK (2016): "Social networks and feelings of social loneliness after migration: The case of European migrants with a native partner in Belgium”, Ethnicities, 16(4), pp. 610-630.

KORNIENKO, O., V. AGADJANIAN, et al. (2018): "Financial and emotional support in close personal ties among Central Asian migrant women in Russia”, Social Networks, 53, pp. 125-135.

LOZARES, C., J. M. VERD y O. BARRANCO (2013): "El potencial analítico de las Redes socio-métricas y ego-centradas: una aplicación al estudio de la Cohesión-Integración de Colectivos sociales", EMPIRIA. Revista de Metodología de Ciencias Sociales, 26, pp. 35-62.

LUBBERS, M. J., J. L. MOLINA, et al. (2010): "Longitudinal analysis of personal networks. The case of Argentinean migrants in Spain", Social Networks, 32, pp. 91104.

LUBBERS, M. J. y J. L. MOLINA (2013): "El proceso de la reconstrucción de la red personal de los inmigrantes: una descripción longitudinal”, EMPIRIA. Revista de Metodología de Ciencias Sociales, 26, pp. 63-88. 
LUBBERS, M. J. y T. A. B. SNIJDERS (2007): “A comparison of various approaches to the exponential random graph model: A reanalysis of 102 student networks in school classes", Social Networks, 29, pp. 489-507.

LUBBERS, M. J., J. L. MOLINA y C. MCCARTY (2007): "Personal Networks and Ethnic Identifications: The Case of Migrants in Spain", International Sociology, 22, pp. 721-741.

MACDONALD, J. S. y L. D. MACDONALD (1964): "Chain Migration, Ethnic Neighborhood Formation and Social Networks", Milbank Memorial Fund Quaterly, 42(1), pp. 82-97.

MARTÍNEZ GARCÍA, M. F., M. GARCÍA RAMÍREZ e I. MAYA JARIEGO (2001): "Una tipología analítica de las redes de apoyo social en inmigrantes africanos en Andalucía”, REIS, 95, pp. 99-125.

MARTÍNEZ GARCÍA, M. F., M. GARCÍA RAMÍREZ e I. MAYA JARIEGO (2002): "Social support and locus of control as predictors of psychological well-being in Moroccan and Peruvian immigrant women in Spain", International Journal of Intercultural Relations, 26, pp. 287-310.

MASSEY, D. S., J. ARANGO, G. HUGO et al. (1993): “Theories of International Migration: A Review and Appraisal", Population and Development Review, 3, pp. 431-466.

MAYA JARIEGO, I. (2002): “Tipos de redes personales de los inmigrantes y adaptación psicológica", Revista Redes, 1(4).

MAYA JARIEGO, I. (2004): "La formación de comunidades de inmigrantes: desplazamiento en cadena y contexto de recepción", Araucaria. Revista Iberoamericana de Filosofía, Política y Humanidades, 12.

MAYA JARIEGO, I. (2006): "Mallas de paisanaje: el entramado de relaciones de los inmigrantes". En: J. L. Pérez Pont, Geografías del desorden. Migración, alteridad y nueva esfera social, Valencia, Universidad de Valencia, pp. 257-276.

MAYA-JARIEGO, I. (2016): "7 usos del análisis de redes en la intervención comunitaria”, REDES. Revista Hispana para el Análisis de Redes Sociales, 27, (2), 1-10.

MAYA-JARIEGO, I. y N. ARMITAGE (2007): "Multiple Senses of Community in Migration and Commuting. The Interplay between Time, Space and Relations", International Sociology, 22(6), pp. 743-766.

MCCARTY, C. (2002): "Structure in personal networks", Journal of Social Structure, 3(1).

MIGUEL LUKEN, V. DE; CARVAJAL GUTIÉRREZ, C. (2007): "Percepción de la inmigración y relaciones de amistad con los extranjeros en los institutos", Revista Migraciones, 22, pp. 147-190.

MIGUEL LUKEN, V. DE; M. J. LUBBERS; M. SOLANA SOLANA; D. RODRÍGUEZ GARCÍA, D. (2015): "Evaluación de la integración social de la población extranjera en Cataluña a partir de las uniones con españoles. Avances desde una perspectiva metodológica mixta", REIS, 150, pp. 151-172.

MIGUEL LUKEN, V. DE; M. SOLANA SOLANA; À. PASCUAL DE SANS (dir.) (2007): Redes sociales de apoyo. La inserción de la población extranjera, Bilbao, Fundación BBVA.

MIGUEL LUKEN, V. DE; M. TRANMER (2010): "Personal support networks of immigrants to Spain: A multilevel analysis", Social Networks, 32(4), pp. 253-262.

MOLINA, J. L. (2005): "El estudio de las redes personales: contribuciones, métodos y perspectivas", EMPIRIA. Revista de Metodología de Ciencias Sociales, 10, pp. 71106. 
MOLINA, J. L., J. LERNER y S. GÓMEZ MESTRES (2008): "Patrones de cambio de las redes personales de inmigrantes en Cataluña", REDES- Revista hispana para el análisis de redes sociales, 15(4).

MOLINA, J. L., M. BOLÍBAR, et al. (2011): "La dispersión geográfica de las redes personales: cálculo y significado", REDES- Revista hispana para el análisis de redes sociales, 20, pp. 113-131. Recuperado de: http://www.redalyc.org/articulo. oa? id=93122194005

PEDONE, C. (2003): “Tú siempre jalas a los tuyos". Cadenas y redes migratorias de las familias ecuatorianas hacia España, Departament de Geografia, Barcelona, Universitat Autònoma de Barcelona.

POPIELARZ, P. A. y T. CSERPES (2018): “Comparing the discussion networks and voluntary association memberships of immigrants and non-immigrants in U.S. suburban gateways", Social Networks, 53, pp. 42-56.

PORTES, A. (2013): Sociología económica. Una investigación sistemática, Madrid, CIS.

PORTES, A. y J. BÖRÖCZ (1989): "Contemporary Immigration: Theoretical Perspectives On Its Determinants And Modes Of Incorporation”, International Migration Review, 23(3), pp. 606-630.

PORTES, A. y J. SENSENBRENNER (1993): "Embeddedness and Immigration: Notes on the Social Determinants of Economic Action", American Journal of Sociology 98(6), pp. 1320-50.

PUTNAM, R.D. (2000): “Bowling Alone: America's Declining Social Capital”. En: CROTHERS L., LOCKHART C. (eds) Culture and Politics, Palgrave Macmillan, New York.

REPKE, L., y V. BENET-MARTÍNEZ (2018): “The (Diverse) Company You Keep: Content and Structure of Immigrants' Social Networks as a Window into Intercultural Relations in Catalonia”, Journal of Cross-Cultural Psychology, 49(6), pp. 924-944.

REQUENA SANTOS, F. (1989): “El concepto de red social”, REIS, 48, pp. 137-152.

REQUENA SANTOS, F. (1996): Redes sociales y cuestionarios, Madrid, CIS.

REQUENA SANTOS, F. (2008): Redes sociales y sociedad civil, Madrid, CIS.

REY POVEDA, A. DEL y D. VONO DE VILHENA (2014): "Marrying after arriving: The role of individuals' networks for immigrant choice of partner's origin", Advances in Life Course Research, 19, pp. 28-39.

RIPLEY, R. M. y T. A. B. SNIJDERS (2018): Manual for RSiena. University of Oxford: Department of Statistics; Nuffield College; University of Groningen: Department of Sociology: 245. Disponible en: https://www.stats.ox.ac.uk/ snijders/siena/RSiena Manual.pdf. Recuperado el 25/10/18.

RODRÍGUEZ GARCİA, D.; M. SOLANA SOLANA; V. DE MIGUEL LUKEN; M. J. LUBBERS (2014): "Immigració, unions mixtes i integració sociocultural a Catalunya: cap a una anàlisi complexa multi-mètode", Documents d'Anàlisi Geogràfica, 60(3), pp. 627-657.

RUIZ-SANTACRUZ, J. S. (2019): "Sistemas migratorios latinoamericanos y desarrollo humano: el alcance de usar el análisis de redes sociales", REDES- Revista hispana para el análisis de redes sociales, 30(1), pp. 79-87.

SNIJDERS, T. A. B. (2005): "Models for Longitudinal Network Data". En: P. CARRINGTON, J. SCOTT y S. WASSERMAN (eds.), Models and Methods in Social Network Analysis, Cambridge, Cambridge University Press. 
STEGLICH, C., T. A. B. SNIJDERS, et al. (2010): "Dynamic Networks and Behavior: Separating Selection from Influence”, Sociological Methodology, 40(1), pp. 329393.

VACCA, R., G. SOLANO, et al. (2018): "A personal network approach to the study of immigrant structural assimilation and transnationalism", Social Networks, 53, pp. 72-89.

VAN DUIJN, M. A. J., J. T. V. BUSSCHBACH, et al. (1999): "Multilevel analysis of personal networks as dependent variables", Social Networks, 21, pp. 187-209.

VERD, J. M.; BOLÍBAR, M.; MARTÍ, J. (2017): "La evolución de las redes personales de la población inmigrada en Cataluña. Un análisis desde los métodos mixtos", QUIT Working paper series, 22. http://ddd.uab.cat/record/179898.

VIRY, G. (2012): "Residential mobility and the spatial dispersion of personal networks: Effects on social support", Social Networks, 34, pp. 59-72. doi:10.1016/j.socnet.2011.07.003

VONO DE VILHENA, D. y E. VIDAL-COSO (2012): "The impact of informal networks on labour mobility: Immigrants' first job in Spain”, Migration Letters, 9(3), pp. 237-247.

WASSERMAN, S. y K. FAUST (2013): Análisis de redes sociales. Métodos y aplicaciones, Madrid, CIS. 